\title{
Una experiencia de aprendizaje servicio interciclo: Relaciones interorganizacionales y orientación de carrera.
}

\author{
Tordera, N. ${ }^{\text {, }}$ Martínez, E. ${ }^{\text {b }}$, Silla, I. ${ }^{\text {a }}$, Salvo, H-X ${ }^{\text {, }}$ Raga, F. ${ }^{\text {b }}$ \\ a IDOCAL. Universitat de València, nuria.tordera@uv.es; inmaculada.silla@uv.es; \\ SCOOP, empar@escolagavina.cat; hectors@escolagavina.cat; praga@escolagavina.cat
}

\begin{abstract}
This service learning collaboration combines community service with academic learning. With that purpose, a collaboration network was created between the University of Valencia and Escola Gavina, a preschool, primary and secondary school. The objective was twofold, first, it attempts to give postgraduate students the opportunity to carry out a diagnostic of the inter-organizational relations of Escola Gavina. This objective is pursued through collaboration between different entities, which is one of the basis of service learning. Inter-organizational relationships are crucial in today's society, they are becoming more frequent, and allow some organizations to achieve objectives that would not otherwise be possible. Second, it allows to provide academic and professional orientation to students of secondary school, while stimulating the development of linguistic competences, autonomy, and social interaction. Twenty students from the master's degree and twenty-seven from secondary school participated in the study. The learning process, their self-perceived competence acquisition, and their degree of satisfaction were assessed. In general, the results showed that this collaboration between students from different educational cicles achieved the intendeed objectives.
\end{abstract}

Keywords: Service learning, interorganizational relationships, collaboration between different educational levels.

\section{Resumen}

Este trabajo es una experiencia de aprendizaje servicio que combina el servicio a la comunidad con el aprendizaje académico. Para ello, se crea una red de colaboración entre la Universitat de València y Escola Gavina, colegio de educación infantil, primaria y secundaria. El objetivo es doble, por un parte, persigue dar a los estudiantes de postgrado la oportunidad de llevar a cabo un diagnóstico de las relaciones interorganizacionales de Escola Gavina. En la actualidad, las relaciones interorganizacionales son cada vez más frecuentes, y permiten que algunas organizaciones alcancen objetivos que de otro modo no sería posible. Además, este objetivo se persigue a través de la colaboración entre diferentes entidades que es una de las bases del aprendizaje servicio. Por otra parte, permite proporcionar orientación académica y profesional a los estudiantes de $4^{\circ}$ de ESO a la vez que se estimula el desarrollo de compentencias lingüisticas, de autonomía, e interacción social. En el estudio participaron 20 estudiantes del máster y 27 alumnos de $4^{a}$ de ESO. Se evaluó el proceso de aprendizaje, su autopercepción de la adquisición de competencias, y el grado de satisfacción con la actividad desarrollada. En general, los resultados muestran que la presente experiencia de innovación y colaboración educativa interciclo consiguió los objetivos que pretendía.

Palabras clave: Aprendizaje servicio, , relaciones interorganizacionales, colaboración niveles educativos. 


\section{Introducción}

El aprendizaje servicio combina el servicio a la comunidad y el aprendizaje académico. Ambos aspectos son igualmente relevantes manteniendo un estrecho equilibrio. A su vez, el aprendizaje servicio crea vínculos sociales, y fortalece el compromiso social y el sentido de responsabilidad cívica de los estudiantes (Martín y Puig, 2017). Cabe destacar que esta metodología de aprendizaje permite la integración de las tres misiones de la universidad: docencia, extensión o responsabilidad social, e investigación (Nieves, 2008). Además, contribuye a generar espacios de transformación social.

La experiencia de aprendizaje servicio que se describe en este trabajo responde al objetivo de desarrollo sostenible "4. Educación de calidad". La colaboración entre el alumnado de un máster Erasmus Mundus y el de $4^{\circ}$ de la ESO contribuye a la formación en lenguas extranjeras y la orientación académica y profesional de estos últimos, a la vez que permite el aprendizaje de los alumnos de postgrado sobre las relaciones interorganizacionales y las organizaciones en red.

\subsection{Aprendizaje servicio}

Nieves (2008) define el aprendizaje servicio como "una actividad o programa de servicio solidario protagonizado por los estudiantes, orientado a atender eficazmente necesidades de una comunidad, y planificada de forma integrada con los contenidos curriculares con el objetivo de optimizar los aprendizajes" (p. 43). De este modo, contribuye al desarrollo de la Universidad como un espacio de aprendizaje ético en el que se estimula el compromiso y la responsabilidad social a través de la participación activa en iniciativas que contribuyen a la mejora de la sociedad (CADEP, 2015). Esta metodología de aprendizaje se diferencia de otras iniciativas como el voluntariado, o el trabajo de campo, porque en el caso del aprendizaje servicio tanto el aprendizaje como el servicio a la comunidad son igualmente prioritarios.

El aprendizaje servicio se basa en el trabajo en red, en este caso, se establecen alianzas entre entidades de diferentes ciclos educativos. Además, dado que el objeto de estudio son las relaciones interorganizacionales, esta iniciativa pone a los estudiantes en contacto con otras comunidades y sus necesidades, favoreciendo la educación en valores y el compromiso social.

\subsection{Las relaciones interorganizacionales}

En el contexto organizacional actual cada vez es más frecuente el establecimiento de redes de relaciones interorganizacionales en el que organizaciones que son independientes legalmente pero que comparten intereses y características convergentes, pero también divergentes, se conectan entre sí a través de relaciones de intercambio recíprocas e interactivas (Van Gils, 1998). Dichas colaboraciones permiten a las organizaciones conseguir objetivos que de otro modo serían difícilmente alcanzables bien por razones de tipo económico (elevados costes de transacción o adquisición) o por razones de tipo social (falta de legitimidad). Por ello, es importante que los estudiantes del Máster Erasmus Mundus en Psicología del Trabajo y las Organizaciones (WOP-P) adquieran las competencias necesarias para hacer un diagnóstico sobre el funcionamiento de estas redes y la consecución de sus objetivos.

Por este motivo se estableció colaboración con la cooperativa Escola Gavina. En el caso de las cooperativas la colaboración con otras organizaciones forma parte además de los principios básicos de funcionamiento definidos por la Asociación Internacional de cooperativas (en inglés ICA, 2015). Cabe destacar que la actividad de innovación docente no sólo permite analizar y evaluar el funcionamiento de las redes interorganizacionales, sino que además lo hace a través de la propia creación de un acuerdo de colaboración interorganizacional. De este modo, los estudiantes aprenden en un entorno real, practicando lo que a su vez es el objeto de estudio. 


\subsection{Contextualización de la innovación}

En este proyecto de colaboración en aprendizaje servicio participan el Instituto de Investigación en Psicología de los RRHH, del Desarrollo Organizacional y de la Calidad de Vida Laboral (IDOCAL, Universitat de València) y Escola Gavina SCOOPV, colegio de educación infantil, primaria y secundaria. Así pues, este proyecto supone la colaboración en red de profesorado y estudiantes de distintos ciclos formativos. En concreto participan 20 alumnos del Máster Erasmus Mundus en Psicología del Trabajo y las Organizaciones de la Universitat de València (enseñanza universitaria de postgrado en psicología) y 27 alumnos de cuarto curso de enseñanza secundaria obligatoria de Escola Gavina.

Las características que hacen idónea la colaboración entre el Máster Erasmus Mundus WOP-P y Escola Gavina son, por un lado, el carácter internacional del máster y el hecho de que Escola Gavina es una cooperativa con una amplia red de colaboración con otras organizaciones. A continuación se describe más en detalle de qué manera esta colaboración satisface las necesidades de aprendizaje de los estudiantes de postgrado al tiempo que ofrece una oportunidad de orientación académica y profesional al alumnado de $4^{\circ}$ de la ESO.

El Máster Erasmus Mundus en Psicología del Trabajo y las Organizaciones (WOP-P) es el único programa Erasmus Mundus en el área de psicología en Europa. Se trata de un consorcio de cuatro universidades europeas que están impartiendo este programa: Universitat de València, Universitat de Barcelona, Alma Mater Studiorum-Universitá di Bologna y Universidade de Coimbra. Se imparten un total de 120 créditos ECTS en dos años y una de las áreas de aprendizaje de este programa es la Psicología de las Organizaciones, disciplina orientada al estudio y la intervención sobre la conducta colectiva en el seno de las organizaciones.

Por otro, cabe mencionar que Escola Gavina es una empresa cooperativa de trabajo asociado, cuya actividad principal es la enseñanza. Se trata de un centro concertado formado por personal contratado y socios cooperativistas (28), que o bien son maestros, o personal de administración y servicios (PAS). También existe la figura del asociado, normalmente antiguos socios que al jubilarse mantienen su capital social en el proyecto y tienen derecho a recibir información sobre lo que hacemos, pero no tienen derecho a votar.

Escola Gavina establece relaciones pedagógicas con otras organizaciones, con las familias, y con los alumnos. Además, ha creado vínculos y alianzas con otras entidades con las que comparten principios pedagógicos y económicos.

Entre los diversos objetivos formativos que persigue el Máster Erasmus Mundus WOP-P está el de desarrollar las competencias de sus estudiantes en el conocimiento de las relaciones interorganizacionales y organizaciones en red con una perspectiva centrada en los grupos de interés. Resulta de especial relevancia para este objetivo el conocimiento de diversos tipos de empresas y especialmente empresas cooperativas con una larga tradición de colaboración con diversos tipos de organizaciones e instituciones, como es el caso de Escola Gavina, una cooperativa valenciana de educación.

En el programa de enseñanza de los alumnos de $4^{\circ}$ de ESO de Escola Gavina, una parte importante para su desarrollo posterior es la formación en lenguas extranjeras y la orientación académica y profesional. Dentro de los programas de orientación, el contacto con jóvenes que han finalizado los grados universitarios y con procedencias internacionales diversas, como es el caso de los alumnos del Máster Erasmus Mundus WOP-P, contribuye a sus objetivos de formación y de orientación. Al mismo tiempo, sirve para estimular el aprendizaje de otras lenguas y especialmente el inglés (idioma oficial del máster).

(c) EY-NC-No 2021, Universitat Politècnica de

València Congreso In-Red (202ロ) 


\section{Objetivos}

En este contexto se establece una alianza de colaboración entre ambas entidades con el objetivo de desarrollar diversas competencias previstas en su plan docente. Este proyecto de aprendizaje servicio tiene un doble objetivo:

- Objetivo 1: Posibilitar que los estudiantes de postgrado realicen un diagnóstico de las relaciones interorganizacionales de Escola Gavina con otras organizaciones. Esta actividad se lleva a cabo en un entorno real y a través del desarrollo de una alianza de colaboración, que a su vez es el objeto de estudio.

Dicho de otro modo, los propios estudiantes participan en la creación de aquello que estudian. Así, los estudiantes de postgrado participan en un proceso de aprendizaje que, a la vez que les permite aprender sobre las relaciones interorganizacionales, les impele a trabajar sobre necesidades reales de dichas organizaciones. Además, el diagnóstico les permite proponer soluciones y mejorar dichas necesidades organizativas.

- Objetivo 2: Proporcionar orientación académica y profesional a los estudiantes de $4^{\circ}$ de ESO y estimular el desarrollo de competencias lingüísticas, de autonomía personal e interacción social.

\section{Desarrollo de la innovación}

El aprendizaje servicio, junto con el aprendizaje cooperativo y por proyectos, están en el núcleo de esta experiencia de colaboración. Los estudiantes del Máster WOP-P trabajan en pequeños grupos con el objetivo de realizar un diagnóstico de las relaciones interorganizacionales entre la cooperativa Escola Gavina y otras cooperativas. Estos grupos tienen la responsabilidad de recabar información, diseñar un plan de evaluación, llevar a cabo entrevistas y un análisis y propuesta de evaluación. Para ello, se establecen varias sesiones de trabajo entre las que se incluye una en la que interactúan las dos entidades que forman parte de la colaboración.

En el caso de los estudiantes de Escola Gavina, la actividad está coordinada entre los tutores del grupo de $4^{\circ}$ de eso y la directora pedagógica del centro. La colaboración con IDOCAL (Universitat de València) se enmarca dentro del bloque de orientación académica y profesional del plan de acción tutorial correspondiente y busca, además, fomentar el desarrollo de competencias lingüísticas, de autonomía personal, y de interacción social. Los alumnos preparan una entrevista que realizarán en inglés a los estudiantes del Master WOP-P y que se que organizan en dos bloques: por una parte, las que tienen relación con su itinerario formativo y, por otra, aquellas que les permiten conocer sus países de origen.

La interacción que permite poner en contacto a los estudiantes de los diferentes ciclos formativos se realiza fundamentalmente a lo largo de una mañana en la que los estudiantes del Master WOP-P visitan las instalaciones de Escola Gavina y se divide en dos grandes etapas:

- Primera etapa. El equipo directivo y el consejo rector de la cooperativa de enseñanza Escola Gavina participa en el proceso de enseñanza de los alumnos del Máster WOP-P a través de las siguientes acciones: facilitación de documentación sobre la cooperativa y su red de relaciones interorganizacionales (antes de la visita), visita guiada a las instalaciones del colegio, sesión de presentación del colegio a los alumnos del Master WOP-P, entrevista de los alumnos del máster con el equipo directivo y la presidencia como parte de un trabajo de clase de la asignatura Organizational Psychology and Organizational Behavior. De esta manera los estudiantes tienen la oportunidad de conocer de primera mano las principales características de la escuela cooperativa y del cooperativismo en general, así como hablar directamente con aquellas personas que están implicadas en el establecimiento de varias redes de colaboración (AKOE, Committee Europa, FEVECTA ...).

2021, Universitat Politècnica de

València Congreso In-Red (202ロ) 
- Segunda etapa. Sesión de intercambio entre los alumnos de $4^{\circ}$ de ESO de la Escola Gavina y los alumnos del Máster WOP-P, en la que los alumnos de secundaria entrevistan a los alumnos de postgrado sobre su desarrollo de carrera, las decisiones de hacer estudios en el extranjero y las similitudes y diferencias entre los distintos sistemas de educación de procedencia. Para llevar a cabo la actividad se separa la clase en dos grupos (10 alumnos aproximadamente). En cada uno de ellos participan alumnos del Master WOP-P procedentes de distintas zonas geográficas (Europa, Asia, América ...). Una vez divididos, los alumnos de la ESO formulan las preguntas que han preparado con antelación con los tutores y los profesores de inglés. Posteriormente se dividen los dos grupos en grupos más pequeños ( 3 o 4 alumnos) para permitir una conversación más informal y estimular la participación de todo el alumnado.

\section{Resultados}

En general se realiza una valoración muy positiva de la colaboración desarrollada así como de los resultados alcanzados tanto por parte del Máster como de Escola Gavina.

Para llevar a cabo la evaluación del proceso de enseñanza aprendizaje se toman en cuenta dos aspectos. Por un lado, se realiza un análisis cualitativo por parte de los docentes que participan en la actividad. En dicho análisis se tiene en cuenta la evaluación del trabajo realizado por los alumnos y los resultados de aprendizaje. Por otro lado se realiza un pase de cuestionarios a los alumnos tanto del Máster WOP-P como de Escola Gavina. Mediante dichos cuestionarios se pretende evaluar tanto la autopercepción de la adquisición de competencias como el grado de satisfacción con la actividad desarrollada.

Durante el proceso de enseñanza aprendizaje se evalúa la adquisición de competencias técnicas y transversales en los alumnos participantes en la actividad en diversos momentos. En primer lugar, antes de que tenga lugar la actividad de intercambio se realizan tutorías específicas con los distintos grupos de trabajo en los que se evalúan los siguientes aspectos: comprensión de la actividad por parte del alumnado, revisión del objetivo propuesto por cada grupo de trabajo para la realización de la actividad, evaluación de los criterios utilizados para la elaboración de la entrevista y la adecuación de las entrevistas para llevar a cabo los procesos de evaluación y diagnóstico. Durante el desarrollo de la actividad se lleva a cabo un seguimiento de la participación de los alumnos en la actividad y la formulación final de las preguntas. Finalmente se evalúa el trabajo final presentado por los alumnos. A cada grupo de trabajo se le proporciona un feedback específico sobre el grado de consecución de los objetivos de enseñanza aprendizaje. Los resultados de aprendizaje alcanzados se valoran de manera muy positiva. Todos los grupos consiguen desarrollar una herramienta de evaluación que cumple con los criterios de evaluación: está fundamentada en la teoría, incluye criterios relevantes para identificar el tipo de relación interorganizacional y las causas de la misma, analiza tanto los aspectos de gestión y funcionamiento como los aspectos más relacionales. Asimismo, en gran medida, el análisis cualitativo de la información obtenida durante el proceso de entrevista permite responder a las cuestiones planteadas para realizar un diagnóstico sobre las relaciones interorganizacionales. Los estudiantes se sienten motivados con la actividad y en general hay un clima positivo y de buena disposición al respecto. No obstante, la tarea reviste la complejidad de haber de enfrentarse a una situación real en la que ellos mismos deben diseñar el proceso de evaluación y deben hacerlo con un equipo de trabajo que ha sido asignado por la profesora, todo lo cual también genera cierta ansiedad.

Una vez finalizada la actividad y el proceso de evaluación y feedback de la profesora se lleva a cabo un pase de cuestionarios a los alumnos en el que como se ha indicado anteriormente se formulan dos tipos de cuestiones sobre autopercepción de competencias adquiridas y satisfacción con la actividad.

Respecto a las preguntas dirigidas a la autopercepción de competencias adquiridas, estás se evalúan con una escala del 1 al 4 que pretende analizar el grado en que el alumno percibe que la metodología utilizada

(c) BY-NC-No 2021, Universitat Politècnica de

València Congreso In-Red (202ロ) 
permite la adquisición de dichas competencias. En 11 de los 17 aspectos analizados la media es superior a 3. En el resto oscila entre 2,6 (la puntuación más baja) y 2,9). Los dos aspectos más valorados fueron la capacidad para desarrollar entrevistas con clientes y directivos para identificar necesidades y problemas y la capacidad para desenvolverse en situaciones multiculturales $(M=3,4)$, seguido por la capacidad para analizar la realidad desde distintas perspectivas teóricas $(\mathrm{M}=3,3)$, recordar aspectos clave del curso $(M=3,2)$, aplicar el conocimiento adquirido a distintas situaciones $(M=3,2)$, diagnosticar la realidad de la organización y desarrollar competencias de comunicación con otros ( $M=3,1)$. Por el contrario, los aspectos peor valorados fueron: diseñar intervenciones fundamentadas teóricamente y capacidad para gestionar tus propias emociones $(M=2,6)$.

Así pues, se pone de manifiesto que los estudiantes perciben que la actividad les ha permitido desarrollar diversas competencias técnicas tanto de análisis como de evaluación o de comprensión de la realidad (Bloom Engelhart, Fürst, Hiss, y Krathwohl, 1956), aunque observan más dificultades para el desarrollo de las competencias de más alto nivel (creación). Por otro lado, también perciben que la actividad fomenta el desarrollo de competencias transversales relacionadas con habilidades sociales y de trabajo en grupo. En este caso, aunque consideran que ha contribuido a mejorar su competencia de comunicación, trabajo multicultural y gestión de relaciones con el entorno, se percibe un desarrollo más limitado de las competencias de gestión emocional.

La satisfacción con la actividad desarrollada en términos globales y con aspectos específicos se analiza con una escala tipo Likert con 5 anclajes de respuesta, en el que 5 es la valoración más positiva y 1 la más negativa. Para los alumnos del Master WOP-P en general la experiencia se evalúa de forma altamente satisfactoria $(\mathrm{M}=4,1)$. Cuando entramos a valorar aspectos más específicos de la actividad los aspectos más valorados fueron: las oportunidades para interactuar con las personas del centro $(M=4,9)$ y tener contacto con la comunidad $(\mathrm{M}=4,6)$, así como el tiempo dedicado a la tarea, el reconocimiento del trabajo realizado y la oportunidad para poner en práctica las propias habilidades y para aprender nuevos conocimientos $(\mathrm{M}=4,1 \mathrm{y} \mathrm{M}=3,9)$. El aspecto peor valorado, aunque no se valora de forma negativa, fue la libertad para usar tu propio método de trabajo $(\mathrm{M}=3,4)$.

Para evaluar el grado de satisfacción y de aprendizaje entre los alumnos de la Escola Gavina, se elabora una encuesta con el objetivo de evaluar la adquisición de competencias lingüística, personales y sociales, así como en qué medida consideran que han recibido información nueva y de utilidad para su orientación educativa y profesional, en una escala de 1 a 5 , siendo 5 el grado máximo de satisfacción o de aprendizaje.

En cuanto al primer bloque de preguntas, tanto el grado de implicación en la elaboración de la entrevista $(\mathrm{M}=4,1)$, de comprensión de lo explicado por los alumnos del máster WOP-P $(\mathrm{M}=4,5)$ y por los propios compañeros $(M=4.4)$ es alto. Además, se han autoevaluado en cuanto al uso del inglés $(M=3,6)$, a ser capaces de vencer el temor de hablar en este idioma $(M=3,7)$ y la mejora de su conocimiento tras la actividad $(\mathrm{M}=2,3)$. En cuanto a las competencias personales, la encuesta permite reflexionar respecto a expresarse con libertad y sinceridad $(M=4,2)$, vencer miedos y vergüenza $(M=3,2)$, al grado de atención $(M=4,1)$, de participación $(M=3,8)$ y de creatividad $(M=3,8)$. Los resultados reflejan la diversidad entre los/as alumnos/as.

El segundo bloque de preguntas les han permitido valorar si la actividad les ha ayudado en cuanto a la orientación académica y profesional. Más de la mitad tienen más clara la opción para el próximo curso (15 alumnos/as), que los itinerarios formativos son diversos (21 alumnos/as) y el tiempo invertido variable (18 alumnos/as), y que la formación postuniversitaria es importante (20 alumnos/as). 
El tercer bloque de preguntas ha evaluado el grado de interacción cultural, humana, social que permite romper prejuicios, ver qué tenemos en común con personas de otros países $(M=3,5)$ y tener más información para poder escoger destinos formativos $(M=4,2)$.

El grado de valoración general sobre la actividad es bastante positivo, ya que el 54\% de los alumnos se ha sentido a gusto o muy a gusto mientras aprendía con gente que acababa de conocer; y el $62 \%$ se ha sentido satisfecho con la interacción con los alumnos de la Universitat. El grado de satisfacción de la actividad en su conjunto se sitúa en el 4,3.

Por otro lado, también hemos querido evaluar cualitativamente el proyecto, para lo cual las dos personas encargadas de llevar a término la sesión formativa al alumnado del Master WOP-P han valorado aspectos como la coordinación de la información que aporta Escola Gavina a los alumnos universitarios, la claridad y la utilidad de dicha información, el reto de expresarse en inglés y de adaptarse al auditorio; el grado de atención y de implicación de los alumnos, la propia satisfacción como comunicadores y cómo ven la imagen de Escola Gavina su auditorio. El grado de satisfacción en todos los aspectos es alto, ya que ambos se han coordinado entre ellos y con la dirección del centro, han hecho un proceso de selección y de la adaptación de la información que entienden que ha sido adecuada y comprensible, a juzgar por el feedback de los alumnos. Valoran muy positivamente el reto de expresarse en inglés.

La evaluación realizada permite detectar algunos de los puntos fuertes de la actividad pero también aspectos que convendría mejorar y desarrollar en un futuro.

\section{Conclusiones finales}

En conclusión consideramos que la presente experiencia de innovación y colaboración educativa interciclo ha conseguido en términos generales los objetivos que pretendía. Por un lado, permite a los estudiantes de postgrado realizar un diagnóstico de las relaciones interorganizacionales en un entorno real y experimentando durante el proceso el desarrollo del mismo tipo de relaciones que estudian: una alianza de colaboración. La actividad desarrollada fomenta el trabajo autónomo y la colaboración en grupos. Además, los estudiantes participan en un proceso de aprendizaje que, a la vez que les permite aprender sobre las relaciones interorganizacionales, les impele a trabajar sobre necesidades reales de dichas organizaciones. Durante el proceso de aprendizaje los alumnos contribuyen al desarrollo de la propia organización al menos en dos sentidos. En primer lugar, facilitando inputs sobre la adecuación o no de las relaciones desarrolladas y las posibles necesidades en términos de alianzas y cooperación de futuro. En segundo lugar, prestando un servicio a los estudiantes de segundo ciclo. Así pues, también permite cumplir el segundo objetivo, proporcionar orientación académica y profesional a los estudiantes de $4^{\circ}$ de ESO y estimular el desarrollo de competencias lingüísticas, de autonomía personal e interacción social. Consideramos que esta experiencia es transferible a otros posibles intercambios entre alumnado de distintos niveles, especialmente estudiantes universitarios de carreras en el ámbito de la educación o de estudios empresariales que puedan incorporar en sus curriculums acciones de contacto con organizaciones educativas cooperativas, y que al mismo tiempo puedan aportar inputs de orientación y desarrollo de habilidades sociales, lingüísticas y/o técnicas a los estudiantes de ciclos formativos distintos.

Cabe destacar que la experiencia vivida va más allá del objetivo académico para convertirse en una oportunidad de intercambio de experiencias y de desarrollo personal de los estudiantes. Por un lado, permite al conjunto de estudiantes del Master internacional acercarse al contexto social y cultural valenciano y pasar un día de aprendizaje fuera del aula. Por otro lado, permite a los estudiantes de último curso de educación secundaria establecer una actividad de acogida en el centro para realizar un encuentro con un perfil de estudiantes novedoso y multicultural. Ello les permite acercarse a realidades tan distintas como pueden ser la vida en una gran urbe de la India o un pueblo de los Alpes italianos. 
Una experiencia de aprendizaje servicio interciclo: Relaciones interorganizacionales y orientación de carrera

Por todo ello consideramos que la actividad ha permitido conseguir los objetivos académicos previstos.

\section{Referencias}

BLOOM, B.S. (1956). "Taxonomy of educational objectives: the classification of educational goals" en Engelhart, M. D., Furst, E. J., Hill, W. H., Krathwohl, D. R. Handbook I Cognitive Domain. New York: David McKay.

CADEP (COMITÉ EJECUTIVO Y EL PLENARIO DE LA COMISIÓN DE SOSTENIBILIDAD) (2015). Institucionalización del Aprendizaje-Servicio como estrategia docente dentro del marco de la Responsabilidad Social Universitaria para la promoción de la Sostenibilidad en la Universidad. Documento Técnico aprobado por el CADEP, celebrado en León el día 29 de mayo de 2015.

INTERNATINOAL CO-OPERATIVE ALLIANCE (ICA) (2015).

$<$ https://www.ica.coop/sites/default/files/publication-files/ica-guidance-notes-en310629900.pdf $>$. [Consulta: 21 de febrero de 2020]

MARTÍN, X., PUIG, J. M. (2017). “Aprendizaje-servicio: conceptualización y elementos básicos” en Rubio, L., Escofet, A. Aprendizaje-servicio (ApS): claves para su desarrollo en la Universidad. Barcelona: Consejo Editorial ICE-Octaedro.

NIEVES, M. (2008). "Calidad académica y responsabilidad social: el aprendizaje servicio como puente entre dos culturas" en Martínez, M. Aprendizaje servicio y responsabilidad social de las universidades. Barcelona: Consejo Editorial ICE-Octaedro.

VAN GILS, M.R. (1998). “Interorganizational networks" en Drenth, P.J., Thierry, H., de Wolff, Ch. J. Handbook of work and organizational psychology. East Sussex, UK: Psychology Press. Volume 4, pp. 89-111. 